IP $\rightleftharpoons$ B

\title{
Higroscopicidade da madeira de três espécies florestais deterioradas por térmitas Nasutitermes
}

\author{
Ezequiel Gallio $^{1 *(\mathbb{D})}$, Paula Zanatta ${ }^{1}$ (D), Débora Duarte Ribes ${ }^{1}(\mathbb{D})$, Rafael Beltrame ${ }^{(\mathbb{D})}$, Darci Alberto Gatto ${ }^{1(D)}$ \\ Universidade Federal de Pelotas, Rua Conde de Porto Alegre, 793, Centro, CEP 96010-290, Pelotas, RS, Brasil
}

"Autor correspondente:

egeng.florestal@gmail.com

Termos para indexação:

Eucalipto

Retratibilidade

Qualidade da madeira

Index terms:

Eucalyptus

Shrinkage

Wood quality

Histórico do artigo:

Recebido em 06/11/2017

Aprovado em 07/05/2018

Publicado em 11/07/2018

doi: 10.4336/2018.pfb.38e201701528
Resumo - Este trabalho objetivou avaliar as modificações resultantes do ataque de cupins quanto à higroscopicidade da madeira de Eucalyptus dunnii, E. saligna e Corymbia maculata. Foram utilizados corpos de prova com dimensões de 20 x 20 x $150 \mathrm{~mm}^{3}$ (tangencial $\mathrm{x}$ radial $\mathrm{x}$ longitudinal), sendo avaliado o teor de umidade de equilíbrio $\left(T U_{E}\right)$, as contrações lineares dos planos tangencial e radial $\left(\beta_{\mathrm{T}}\right.$ e $\left.\beta_{\mathrm{R}}\right)$, a anisotropia da contração $\left(\mathrm{CA}_{\beta}\right)$, a absorção de água (AA) e a taxa de absorção de água (TAA) de amostras de madeira sadias e deterioradas por térmitas Nasutitermes sp. Verificou-se que o ataque dos térmitas causou aumento no $\mathrm{TU}_{\mathrm{E}}$ e redução no $\beta_{\mathrm{T}}$, entretanto, $\beta_{\mathrm{R}} \mathrm{e}$ $\mathrm{CA}_{\beta}$ apresentaram variação entre as diferentes espécies. AA e TAA apresentaram alta intensidade de absorção de água nas primeiras horas de imersão, sendo maiores nas amostras do grupo controle em relação às deterioradas. Possivelmente, as variações nos parâmetros relativos à higroscopicidade da madeira das espécies estudadas estão relacionadas ao aumento da porosidade e deterioração, principalmente da celulose e hemiceluloses, afetando diretamente a capacidade da madeira em absorver e perder umidade, comprometendo a sua qualidade.

\section{Wood hygroscopicity of three forestry species deteriorated by Nasutitermes termites}

\begin{abstract}
The objective of this work was to evaluate hygroscopicity modification of Eucalyptus dunnii, E. saligna and Corymbia maculata wood due to termites attack. Using samples measuring $20 \times 20 \times 150 \mathrm{~mm}^{3}$ (tangential $\times$ radial $\times$ longitudinal) the equilibrium moisture content $\left(\mathrm{MC}_{\mathrm{E}}\right)$, linear contractions of the tangential and radial planes $\left(\beta_{\mathrm{T}}\right.$ e $\left.\beta_{\mathrm{R}}\right)$, contraction anisotropy $\left(\mathrm{CA}_{\beta}\right)$, water absorption (AA) and water absorption rate (TAA) of healthy and deteriorated wood by Nasutitermes termites were evaluated. It was verified that the termite attack caused increase in $\mathrm{TU}_{\mathrm{E}}$ and reduction in $\beta_{\mathrm{T}}$. However, $\beta_{\mathrm{R}}$ and $\mathrm{CA}_{\beta}$ presented different behaviors, varying among the different species. AA and TAA presented higher water absorption intensity in the first hours of immersion, being higher in the control group in comparison with the deteriorated samples. Possibly the variations in the parameters related to the wood hygroscopicity of the studied species are correlated to the increase of porosity and deterioration mainly of cellulose and hemicelluloses, directly affecting the wood's ability to absorb and lose moisture, compromising the material quality.
\end{abstract}




\section{Introdução}

Dentre os mais diversificados tipos de materiais, a madeira merece destaque, visto que características tecnológicas diversificadas the conferem qualidades que propiciam seu emprego para as mais variadas finalidades. Os gêneros Corymbia e Eucalyptus apresentam-se como alternativas viáveis para suprir a demanda por parte do mercado madeireiro (Hornburg et al., 2012), principalmente pela qualidade fornecida decorrente das suas propriedades. Além disso, trata-se de matéria prima renovável e sustentável, porém suscetível à deterioração causada por agentes biológicos (Alves et al., 2006).

Considerando os insetos xilófagos, os térmitas ou cupins são responsáveis por grandes danos na madeira, devido à severidade de seus ataques e consequente comprometimento das características tecnológicas desse material, o que inviabiliza sua utilização (Stallbaun et al., 2017). Nesse contexto, Araújo et al. (2016) ressaltam que a utilização adequada da madeira encontra-se diretamente atrelada ao conhecimento das respectivas características tecnológicas, sendo a higroscopicidade uma das principais propriedades físicas.

A retratibilidade da madeira que ocorre em função da perda ou ganho de umidade é um fator limitante no que diz respeito ao uso mais adequado (Moraes Neto et al., 2009). Além disso, as variações dimensionais recorrentes na madeira podem implicar no surgimento de defeitos, como empenamentos e rachaduras, e então comprometer a qualidade e emprego (Borges \& Quirino, 2004).

Pesquisas que associem as modificações na higroscopicidade da madeira em função da deterioração causada por térmitas ou cupins são escassas. Ainda assim, estudos que abordem as variações recorrentes nas propriedades tecnológicas em função da biodeterioração por agentes xilófagos são de fundamental importância para a compreensão da utilização adequada desse material complexo em função de sua qualificação. Portanto, o objetivo deste trabalho foi avaliar a influência da deterioração provocada por térmitas Nasutitermes sp. quanto à higroscopicidade da madeira das espécies Corymbia maculata, Eucalyptus dunnii e E. saligna.

\section{Material e métodos}

Foram utilizadas tábuas de madeira de três espécies florestais (Eucalyptus saligna - 22 anos; E. dunnii - 28 anos; Corymbia maculata - 22 anos). Foram confeccionados 20 corpos de prova para cada espécie (10 - grupo controle +10 - ensaio de biodeterioração) com dimensões de $20 \times 20 \times 150 \mathrm{~mm}$ (tangencial $\mathrm{x}$ radial $\mathrm{x}$ longitudinal), sendo acondicionados em câmara climatizada, a $20{ }^{\circ} \mathrm{C}$ e $65 \%$ de umidade relativa (UR), até o equilíbrio higroscópico de $12 \%$.

Isso possibilitou a determinação de massas e dimensões iniciais, para posteriores cálculos dos parâmetros relacionados à higroscopicidade das amostras de madeira.

\section{Ensaio de biodeterioração com Nasutitermes sp.}

O ensaio foi conduzido por meio da adaptação da norma American Society for Testing and Materials D 3345 (2008), por um período de 40 dias. Postaram-se os corpos de prova em uma caixa de água (capacidade de 2000 L), com o fundo recoberto com uma camada de areia de aproximadamente $15 \mathrm{~cm}$, periodicamente umedecida com água destilada, visando suprir as necessidades para o desenvolvimento dos térmitas.

Ao final do ensaio, os substratos e térmitas remanescentes foram removidos dos corpos de prova com o auxílio de pincel e espátula e, posteriormente, ficaram acondicionados em ambiente com temperatura e umidade controladas $\left(20^{\circ} \mathrm{C}\right.$ e $65 \%$ UR).

\section{Higroscopicidade da madeira}

As variáveis estudadas foram:umidade de equilíbrio, absorção e taxa de absorção de água, contração nos planos anatômicos radial e tangencial e anisotropia. Inicialmente foi determinada a retratibilidade, por meio da contração. A absorção de água na madeira foi efetuada até o instante em que os corpos de prova apresentaram massa constante em pesagens sucessivas, indicando a completa saturação em água, enquanto determinou-se a taxa de absorção referente ao período de $48 \mathrm{~h}$.

As equações empregadas para a determinação das variáveis estudadas estão descritas na Tabela 1.

\section{Análise estatística dos resultados}

Os resultados foram submetidos à análise da variância, sendo as médias comparadas, posteriormente, por meio do teste LSD de Fisher, com 5\% de probabilidade de erro. Considerando a mesma probabilidade, modelos de ajuste $(y=\sqrt{ }[\mathrm{a} \pm(\mathrm{b} * \sqrt{ } \mathrm{x})])$, foram descritos relacionando a absorção de água (AA) e o tempo. Selecionou-se esse modelo com base em testes preliminares, que indicaram para esse modelo o melhor ajuste para os dados analisados. 
Tabela 1. Equações empregadas para determinação de parâmetros relacionados à higroscopicidade das madeiras.

\begin{tabular}{ll}
\hline \multicolumn{1}{c}{ Propriedade } & \multicolumn{1}{c}{ Equação } \\
\hline Umidade de equilíbrio & $\mathrm{TU}_{\mathrm{E}}=\frac{\mathrm{M}_{12}-\mathrm{M}_{0}}{\mathrm{M}_{0}} * 100$ \\
Absorção de água & $\mathrm{AA}=\frac{\mathrm{M}_{\mathrm{U}}-\mathrm{M}_{0}}{\mathrm{M}_{0}} * 100$ \\
Taxa de Absorção de água & $\mathrm{TAA}=\frac{\mathrm{M}_{\mathrm{U}}-\mathrm{M}_{0}}{\Delta \mathrm{t}}$ \\
Tangencial & $\beta_{\mathrm{T}}=\frac{\mathrm{DT}_{\mathrm{S}}-\mathrm{DT}_{0}}{\mathrm{DT}_{\mathrm{S}}} * 100$ \\
Contração $(\beta) \quad \begin{array}{l}\text { Radial } \\
\text { anisotropia }\end{array}$ & $\beta_{\mathrm{R}}=\frac{\mathrm{DR}_{\mathrm{S}}-\mathrm{DR}_{0}}{\mathrm{DR}_{\mathrm{S}}} * 100$ \\
&
\end{tabular}

Em que: $\mathrm{TU}_{\mathrm{E}}=$ teor de umidade de equilíbrio (\%); $\mathrm{AA}=$ absorção de água $(\%) ; \mathrm{TAA}=$ taxa de absorção de água $\left(\mathrm{g} \mathrm{h}^{-1}\right) ; \beta_{\mathrm{T}}=$ contração no plano tangencial $(\%) ; \beta_{\mathrm{R}}=$ contração no plano radial (\%); $\mathrm{CA}_{\beta}=$ coeficiente de anisotropia de contração; $\mathrm{M}_{12}=$ massa da amostra em condição de equilíbrio higroscópico (g); $\mathrm{M}_{0}=$ massa da amostra seca em estufa a $103 \pm 2{ }^{\circ} \mathrm{C}(\mathrm{g}) ; \mathrm{M}_{\mathrm{U}}=$ massa da amostra em condição saturada $(\mathrm{g}) ; \Delta \mathrm{t}=$ variação do tempo de imersão dos corpos de prova $(\mathrm{h}) ; \mathrm{DT}_{\mathrm{S}}=$ dimensão no plano tangencial da amostra em condição saturada $(\mathrm{cm}) ; \mathrm{DT}_{0}=$ dimensão no plano tangencial após secagem em estufa a $0 \%$ de umidade $(\mathrm{cm}) ; \mathrm{DR}_{\mathrm{S}}=$ dimensão no plano radial da amostra em condição saturada $(\mathrm{cm}) ; \mathrm{DR}_{0}=$ dimensão no plano radial após secagem em estufa a $0 \%$ de umidade $(\mathrm{cm})$.

\section{Resultados}

Verificou-se diferença significativa no teor de umidade de equilíbrio das amostras controle em relação às deterioradas somente para Corymbia maculata (Tabela 2). Após o ataque, ocorreram aumentos percentuais dessa propriedade física para todas as espécies: C. maculata (8,8\%), Eucalyptus dunnii (47,8\%) e E. saligna (4,1\%).

Nota-se que nas amostras do grupo controle a contração no plano tangencial é maior em comparação ao plano radial (Tabela 3). A madeira de E. saligna apresenta maior estabilidade dimensional frente às demais (considerando os valores de contração nos planos e a anisotropia). Apenas a contração no plano tangencial foi diferente significativamente, sendo a madeira de $E$. dunnii mais suscetível a defeitos devido à retratibilidade, pois a mesma apresentou a maior anisotropia.
Tabela 2. Valores médios do teor de umidade de equilíbrio para as amostras de madeira controle e as deterioradas por térmitas.

\begin{tabular}{cccc}
\hline \multirow{2}{*}{ Tratamento } & \multicolumn{3}{c}{ Teor de umidade de equilíbrio (\%) } \\
\cline { 2 - 4 } & $\begin{array}{c}\text { Corymbia } \\
\text { maculata }\end{array}$ & $\begin{array}{c}\text { Eucalyptus } \\
\text { dunnii }\end{array}$ & $\begin{array}{c}\text { Eucalyptus } \\
\text { saligna }\end{array}$ \\
\hline Controle & $10,1 \mathrm{a}$ & $10,7 \mathrm{a}$ & $10,7 \mathrm{a}$ \\
Deteriorada & $11,0 \mathrm{~b}$ & $15,9 \mathrm{a}$ & $11,1 \mathrm{a}$ \\
CV (\%) & 4,5 & 35,4 & 4,1 \\
F & $146,9^{*}$ & $3,1^{\mathrm{ns}}$ & $3,0^{\mathrm{ns}}$ \\
\hline
\end{tabular}

Em que: médias nas colunas acompanhadas de mesma letra não diferem entre si, conforme teste LSD Fisher, com 5\% de probabilidade de erro. $\mathrm{CV}=$ coeficiente de variação dos tratamentos; * = significativo a $5 \%$ de probabilidade de erro, segundo o teste $\mathrm{F}(\mathrm{p} \leq 0,05) ;{ }^{\text {ns }}=$ não significativo.

Tabela 3. Valores médios dos parâmetros relacionados à retratibilidade das amostras controle das espécies florestais estudadas.

\begin{tabular}{cccc}
\hline \multirow{2}{*}{ Espécie } & \multicolumn{3}{c}{ Contração- $\boldsymbol{\beta}$} \\
\cline { 2 - 4 } & $\mathbf{T}(\%)$ & $\mathbf{R}(\%)$ & $\mathbf{C A}$ \\
\hline Corymbia maculata & $12,1 \mathrm{~b}$ & $9,7 \mathrm{a}$ & $1,2 \mathrm{a}$ \\
Eucalyptus dunnii & $14,2 \mathrm{c}$ & $9,4 \mathrm{a}$ & $1,6 \mathrm{a}$ \\
Eucalyptus saligna & $10,0 \mathrm{a}$ & $8,3 \mathrm{a}$ & $1,2 \mathrm{a}$ \\
CV (\%) & 17,6 & 16,7 & 24,9 \\
F & $11,0^{*}$ & $1,1^{\text {ns }}$ & $2,2^{\text {ns }}$ \\
\hline
\end{tabular}

Em que: médias nas colunas acompanhadas de mesma letra não diferem entre si, conforme teste LSD Fisher, com $5 \%$ de probabilidade de erro. $\mathrm{T}=$ plano tangencial; $\mathrm{R}=$ plano radial; $\mathrm{CA}=$ coeficiente de anisotropia; $\mathrm{CV}=$ coeficiente de variação dos tratamentos; * = significativo a $5 \%$ de probabilidade de erro, segundo o teste $\mathrm{F}(\mathrm{p} \leq 0,05) ;{ }^{\text {ns }}=$ não significativo.

$\mathrm{O}$ ataque de térmitas Nasutitermes ocasionou variações significativas somente no plano radial e no coeficiente de anisotropia das amostras de madeira de C. maculata (Tabela 4). No que tange à contração no plano tangencial, houve decréscimo para as três espécies: C. maculata (11,2\%), E. dunnii $(3,8 \%)$ e $E$. saligna $(16,2 \%)$.

A contração no plano radial das amostras deterioradas aumentou somente em $E$. dunnii (10,8\%), enquanto as amostras de E. saligna e C. maculata apresentaram reduções de $15,9 \%$ e $23,3 \%$, respectivamente, sendo significativa para a última espécie citada. Quanto à anisotropia, foi observado um aumento significativo para amostras de C. maculata (20,0\%), diferente da redução da anisotropia observada para $E$. dunnii $(28,7 \%)$, não havendo variação para amostras de E. saligna. 
Tabela 4. Resumo estatístico e valores médios de retratibilidade para as amostras de madeira controle e as deterioradas por térmitas.

\begin{tabular}{cccc}
\hline \multicolumn{4}{c}{ Contração no plano tangencial (\%) } \\
\hline Tratamento & $\begin{array}{c}\text { Corymbia } \\
\text { maculata }\end{array}$ & $\begin{array}{c}\text { Eucalyptus } \\
\text { dunnii }\end{array}$ & $\begin{array}{c}\text { Eucalyptus } \\
\text { saligna }\end{array}$ \\
\hline Controle & $12,1 \mathrm{a}$ & $14,2 \mathrm{a}$ & $10,0 \mathrm{a}$ \\
Deteriorada & $10,8 \mathrm{a}$ & $13,6 \mathrm{a}$ & $8,4 \mathrm{a}$ \\
CV (\%) & 13,1 & 25,0 & 16,2 \\
F & $2,3^{\text {ns }}$ & $0,0^{\text {ns }}$ & $3,3^{\text {ns }}$ \\
\hline Plano radial (\%) & 9,4 a \\
\hline Deteriorada & $9,7 \mathrm{~b}$ & $10,4 \mathrm{a}$ & $7,0 \mathrm{a}$ \\
CV (\%) & $7,5 \mathrm{a}$ & 16,4 & 13,6 \\
F & 11,4 & $0,8^{\text {ns }}$ & $1,5^{\text {ns }}$ \\
\hline Controle & $11,5^{*}$ & $1,2 \mathrm{a}$ & $1,6 \mathrm{a}$ \\
\hline Deteriorada & $1,5 \mathrm{~b}$ & $1,1 \mathrm{a}$ & $1,2 \mathrm{a}$ \\
CV (\%) & 12,8 & 34,8 & 4,3 \\
F & $8,9^{*}$ & $2,3^{\text {ns }}$ & $0,0^{\text {ns }}$ \\
\hline
\end{tabular}

Em que: médias nas colunas acompanhadas de mesma letra, para cada parâmetro, não diferem entre si, conforme teste LSD Fisher, com 5\% de probabilidade de erro. $\mathrm{CV}=$ coeficiente de variação dos tratamentos; * = significativo a $5 \%$ de probabilidade de erro, segundo o teste $\mathrm{F}(\mathrm{p} \leq 0,05)$; ${ }^{\text {ns }}$ = não significativo

Pode-se observar pela Figura 1 que o ataque de Nasutitermes sp. provocou variações na absorção (AA) e taxa de absorção de água (TAA).

Quanto à absorção de água para a espécie $C$. maculata (Figura 1A), pode ser observado que a 30\%, passadas $86 \mathrm{~h}$, houve uma mudança no padrão de absorção, sendo que a partir desse ponto as amostras de madeira sadias absorveram maiores quantidades de água em comparação às deterioradas. Foi observado comportamento semelhante para as amostras de $E$. dunnii e E. saligna (Figuras 1C e 1E, respectivamente), com mudança na absorção de água (aproximadamente a $40 \%$ ).

Na Tabela 5 estão apresentados os modelos ajustados para a estimativa da absorção de água do grupo controle e de amostras deterioradas por térmitas, das três espécies florestais. Os modelos apresentaram um ajuste significativo, com elevado valor do coeficiente de determinação ajustado $\left(\mathrm{R}^{2}\right)$, possibilitando estimar satisfatoriamente as variações que ocorrem na absorção de água em função do tempo.
Tabela 5. Modelos ajustados para determinação da absorção de água em função do tempo para madeiras sadias e deterioradas por térmitas Nasutitermes sp.

\begin{tabular}{|c|c|c|c|}
\hline Tratamento & Modelo ajustado & $\begin{array}{c}\mathbf{R}^{2} \\
\text { ajustado }\end{array}$ & $\mathbf{F}$ \\
\hline \multicolumn{4}{|c|}{ Corymbia maculata } \\
\hline Controle & $A A=\sqrt{ }(160,385+(94,2292 * \sqrt{ } T))$ & 0,99 & $4030^{*}$ \\
\hline Deteriorada & $A A=\sqrt{ }(197,095+(81,5179 * \sqrt{ } T))$ & 0,99 & $6609 *$ \\
\hline \multicolumn{4}{|c|}{ Eucalyptus dunnii } \\
\hline Controle & $A A=\sqrt{ }(130,286+(140,427 * \sqrt{ } T))$ & 0,99 & $4027 *$ \\
\hline Deteriorada & $A A=\sqrt{ }(168,315+(130,251 * \sqrt{ } T))$ & 0,99 & $6408 *$ \\
\hline \multicolumn{4}{|c|}{ Eucalyptus saligna } \\
\hline Controle & $A A=\sqrt{ }(175,892+(115,738 * \sqrt{ } T))$ & 0,99 & $6012 *$ \\
\hline Deteriorada & $A A=\sqrt{ }(299,101+(96,0159 * \sqrt{ } T))$ & 0,99 & $4819^{*}$ \\
\hline
\end{tabular}

Em que: $\mathrm{AA}=\mathrm{Absorção} \mathrm{de} \mathrm{água}(\%) ; \mathrm{T}=$ tempo $(\mathrm{h}) ; \mathrm{R}^{2}$ ajustado = coeficiente de determinação ajustado; * = significativo a $5 \%$ de probabilidade de erro, segundo o teste $\mathrm{F}(\mathrm{p} \leq 0,05) ;{ }^{\text {ns }}=$ não significativo.

\section{Discussão}

Possivelmente, o aumento na umidade está associado com a deterioração da parede celular e surgimento de galerias (Clausen, 2010), aumentando a porosidade da madeira, o que possibilita maior fluxo de água em seu interior. Por se tratar de material rico em grupos $\mathrm{OH}$, a quebra das ligações dos componentes primários (celulose e hemicelulose) pode disponibilizar hidroxilas que possuem elevada afinidade com a água (Sendner et al., 2009), ocasionado variações no teor de umidade de equilíbrio da madeira, pela absorção de umidade. A elevação no valor percentual dessa propriedade da madeira representa uma desvantagem, pois permite que elevar sua capacidade de trocar umidade com o ambiente ao qual está exposta, até que se atinja o equilíbrio, dificultando sua secagem e comprometendo assim a qualidade desse material, dependendo do uso a que se destina, devido ao surgimento de defeitos.

Tais defeitos podem estar relacionados à disposição e dimensões dos elementos anatômicos (raios, micelas, fibras) constituintes da madeira, os quais possibilitam distintas variações dimensionais nos planos anatômicos (Oliveira et al., 2010). Segundo Logsdon et al. (2008), madeiras com coeficiente de anisotropia inferior a 1,5 são qualificadas como excelentes, por apresentarem elevada estabilidade dimensional, enquanto madeiras com anisotropia na faixa de 1,5 a 2,0 são consideradas normais. 

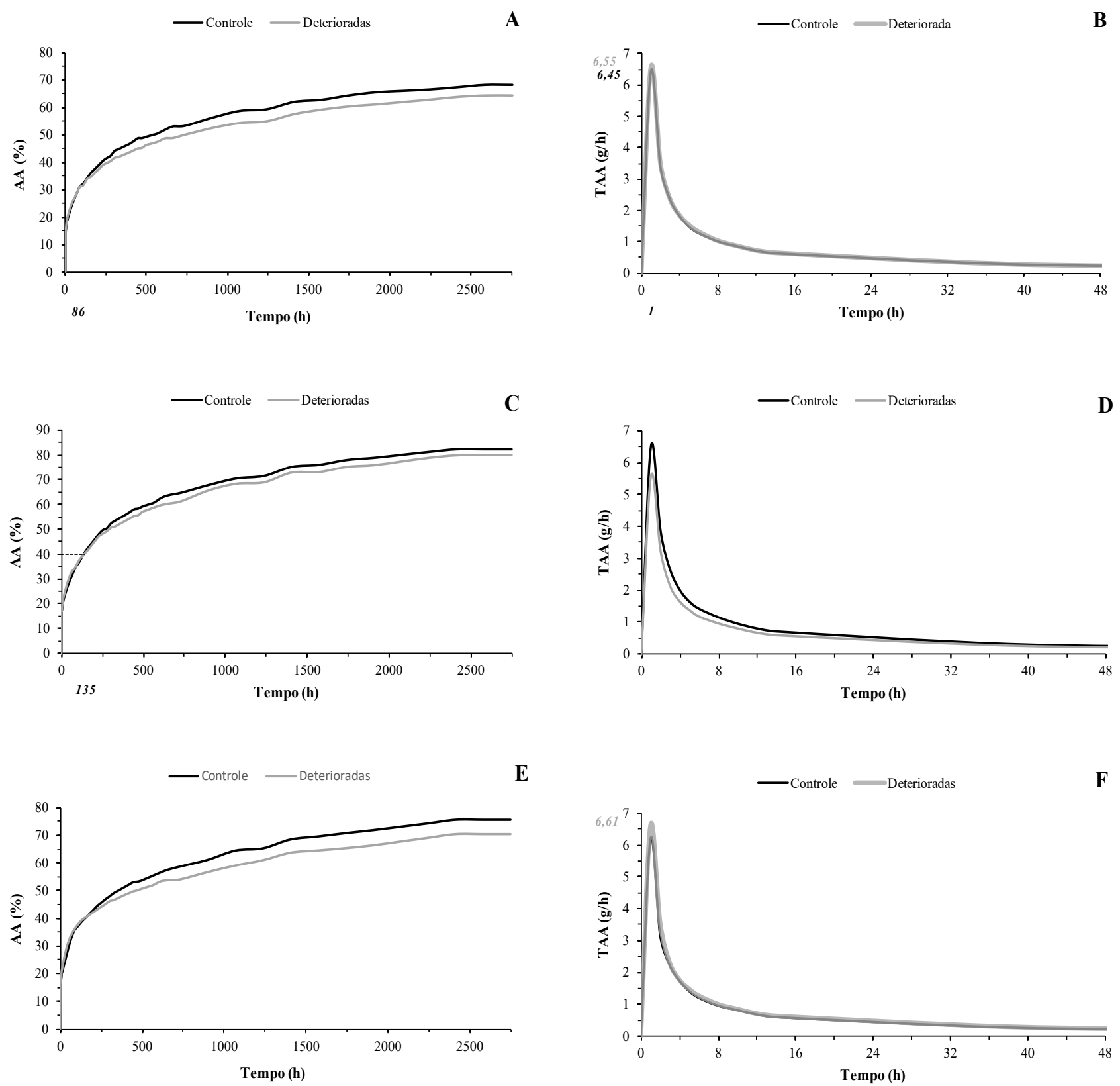

Figura 1. Absorção de água (AA) e taxa de absorção de água (TAA) de amostras de madeira sadias e deterioradas por Nasutitermes sp. de Corymbia maculata (A - B), Eucalyptus dunnii (C - D) e Eucalyptus saligna (E - F).

Em seus estudos, Martins et al. (2013) determinaram uma contração no plano tangencial $(10,4 \%)$ e radial $(8,6 \%)$ inferior à do presente estudo para a madeira de $C$. maculata, porém, com anisotropia semelhante $(1,2)$. Para a madeira de E. dunnii, Batista et al. (2010) obtiveram valores médios superiores para os parâmetros de contração tangencial, contração radial e coeficiente de anisotropia da contração (respectivamente, $12,7 \%, 5,7 \%$ e 2,4\%). Para E. saligna, os mesmos autores determinaram os valores de contração de: 9,9\% (tangencial), 5,0\% (radial) e 2,1 (coeficiente de anisotropia). A discrepância para os parâmetros de retratibilidade encontrados para as espécies de Eucalyptus, em relação aos do presente estudo, podem estar associados à idade dos indivíduos 
(madeiras juvenil e adulta). Em geral, madeiras adultas (mais densas) caracterizam-se por apresentarem paredes mais espessas e lumens menores, tendem a absorver maior quantidade de água por unidade de volume, acarretando em maior contração ou inchamento quando comparadas às de lenho juvenil (Oliveira et al., 2010).

Em geral, as amostras de madeiras deterioradas apresentaram redução dos percentuais de contração em relação ao grupo controle (exceção para $E$. dunnii, no plano radial). A celulose e as hemiceluloses são compostos hidrófilos, ou seja, possuem como característica a fácil adsorção de água (Borges \& Quirino, 2004; Boonstra \& Tjeerdsma, 2006). Possivelmente, essa redução da contração ocorreu devido à decomposição dos sítios (presentes na celulose e hemiceluloses amorfas) que possuem afinidade com a água no interior da madeira, dificultando a absorção de umidade por parte desse material (Hill, 2006). Ou seja, uma vez que os térmitas possuem capacidade de digerir a celulose, a hemicelulose e escarificar a lignina, a redução na quantidade desses componentes ajuda a explicar a maior dificuldade da água em aderir aos radicais $\mathrm{OH}^{-}$e então interferir nas variações dimensionais da parede celular das madeiras.

Com o passar do tempo, devido à redução de sítios de adsorção (ausência dos componentes químicos removidos pelo ataque de Nasutitermes sp.) das amostras deterioradas, a intensidade de absorção de água é reduzida em relação ao grupo controle. Observa-se que a maior intensidade na taxa de absorção de água ocorreu na primeira hora de imersão, devido ao preenchimento dos lumens celulares, por meio de forças de capilaridade, sendo correspondente ao ocorrido na absorção de água. É possível que a mudança no padrão de absorção após $30 \%$ ou $40 \%$ da absorção de água esteja relacionado à menor quantidade de material polimérico nas madeiras deterioradas em relação às amostras do grupo controle. Em seus estudos com dois polímeros distintos (poliestireno e poliuretano), Bouchonneau et al. (2010) também atribuíram a diferença na cinética de absorção de água à redução da quantidade desse material.

\section{Conclusões}

$\mathrm{O}$ ataque de térmitas Nasutitermes sp. causou alterações na higroscopicidade da madeira. A alteração dessa propriedade na madeira exposta a estes insetos é atribuída às modificações nas suas propriedades físicas e anatômicas, pois ao se desenvolverem, aumentam a porosidade e deterioram celulose, hemiceluloses e lignina, as quais possuem caráter hidrofílico, acarretando redução na qualidade desse material e comprometendo sua utilização.

Recomenda-se que em estudos futuros sejam feitas análises que abordem a composição química e sua relação com a higroscopicidade da madeira.

\section{Agradecimentos}

Os autores agradecem à CMPC Celulose RioGrandense pela doação das madeiras e à Coordenação de Aperfeiçoamento de Pessoal do Nível Superior (CAPES), pela concessão da bolsa de doutorado.

\section{Referências}

Alves, M. V. S. et al. Resistência natural de seis espécies de madeiras da região amazônica a fungos apodrecedores, em ensaios de laboratório. Ciência Florestal, v. 16, n. 1, p. 17-26, 2006. DOI: $10.5902 / 198050981884$.

American Society for Testing and Materials. ASTM D 3345 - 74: standard test method for laboratory evaluation of wood and other cellulosic materials for resistance to termites. West Conshohocke, 2008.

Araújo, B. H. P. et al. Propriedades físicas da madeira de Calycophyllum spruceanum Benth. em função do diâmetro e da posição (base e topo) no fuste. Scientia Forestalis, v. 44, n. 111, p. 759-768, 2016. DOI: 10.18671/scifor.v44n111.22.

Batista, D. C. et al. Densidade básica e retratibilidade da madeira de clones de três espécies de Eucalyptus. Ciência Florestal, v. 20, n. 4, p. 665-674, 2010. DOI: 10.5902/198050982425.

Boonstra, M. J. \& Tjeerdsma, B. Chemical analysis of heat treated soft woods. European Journal of Wood and Wood Products, v. 64, p. 204-211, 2006. DOI: 10.1007/s00107-005-0078-4.

Borges, L. M. \& Quirino, W. F. Higroscopicidade da madeira de Pinus caribaea var. hondurensis tratado termicamente. Revista Biomassa \& Energia, v. 1, n. 2, p 173-182, 2004.

Bouchonneau, N. et al. Análise da absorção de água em dois polímeros expandidos: desenvolvimento do módulo de flutuabilidade de um mini-robô submarino. Polímeros, v. 20, n. 3, p. 181-187, 2010. DOI: $10.1590 / \mathrm{S} 0104-14282010005000032$.

Clausen, C. A. Biodeterioration of wood. In: Forest Products Laboratory (United States). Wood handbook: wood as an engineering material. Wisconsin, 2010.

Hill, C. A. S. Wood modification: chemical, thermalandother processes. Chichester: John Wiley \& Sons, 2006.

Hornburg, K. F. et al. Qualidade das toras e da madeira serrada de seis espécies de eucalipto cultivadas no litoral de Santa Catarina. Scientia Forestalis, v. 40, n. 96, p. 463-471, 2012. 
Logsdon, N. B. et al. Caracterização físico-mecânica da madeira de Cedro-marinheiro, Guarea trichilioides L. (Meliaceae). Scientia Forestalis, v. 36, n. 77, p. 43-51, 2008.

Martins, M. et al. Simulação em uso dos pisos de madeira simulação em uso dos pisos de madeira de Eucalyptus sp e Corymbia maculata. Cerne, v. 19, n. 1, p. 151-156, 2013. DOI: 10.1590/S010477602013000100018 .

Moraes Neto, S. P. et al. Propriedades mecânicas da madeira de cinco procedências de Pinus caribaea var. hondurensis implantadas no cerrado do Distrito Federal, DF. Planaltina: Embrapa Cerrados, 2009. 20 p. (Embrapa Cerrados. Boletim de pesquisa e desenvolvimento, 251).
Oliveira, J. T. S. et al. Avaliação da retratibilidade da madeira de sete espécies de Eucalyptus. Revista Árvore, v. 34, n. 5, p. 929-936, 2010. DOI: 10.1590/S0100-67622010000500018.

Sendner, C. et al. Interfacial water at hydrophobic and hydrophilic surfaces: slip, viscosity, and diffusion. Langmuir, v. 25, n. 18, p. 10768-10781, 2009. DOI: 10.1021/la901314b.

Stallbaun, P. H. et al. Resistência natural da madeira de Sclerolobium paniculatum Vogel a cupins em condições de laboratório. Floresta e Ambiente, v. 24, e20160013, 2017. DOI: 10.1590/21798087.001316 . 